
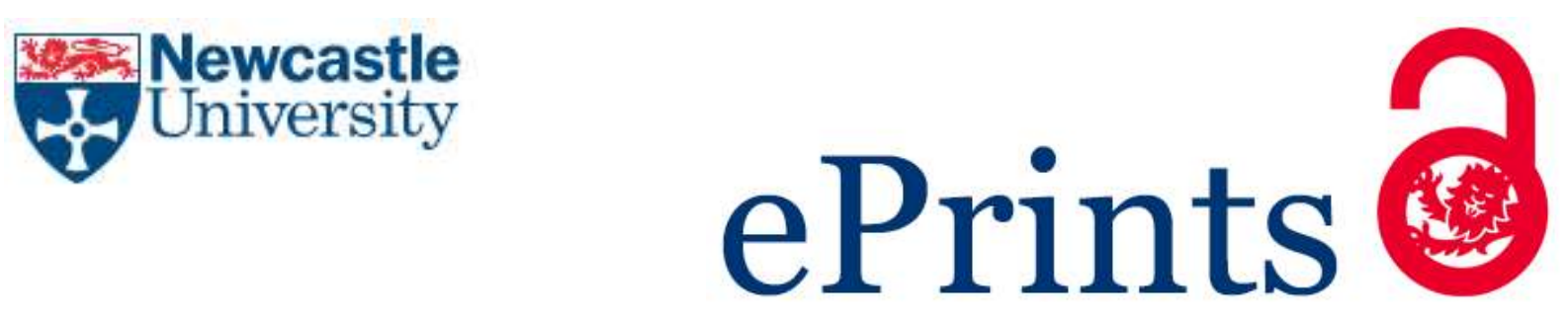

Godfrey A, Morris R, Hickey A, Del Din S. Beyond the front end: investigating a thigh worn accelerometer device for step count and bout detection in Parkinson's disease. Medical Engineering \& Physics 2016, 38(12), 1524-1529. Copyright:

(C) 2016. This manuscript version is made available under the CC-BY-NC-ND 4.0 license DOI link to article:

http://dx.doi.org/10.1016/i.medengphy.2016.09.023

Date deposited:

$23 / 11 / 2016$

Embargo release date:

22 October 2017

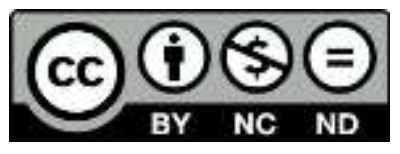

This work is licensed under a

Creative Commons Attribution-NonCommercial-NoDerivatives 4.0 International licence 


\title{
Beyond the front end: investigating a thigh worn accelerometer device for step count and bout detection in Parkinson's disease
}

\author{
A. Godfrey ${ }^{1,2}$, R. Morris ${ }^{1,2}$, A. Hickey ${ }^{1,2}$, S. Del Din $^{1,2}$ \\ ${ }^{1}$ Institute of Neuroscience | Newcastle University Institute for Ageing, Newcastle University, \\ Newcastle upon Tyne, UK
}

\section{Corresponding author}

Dr Alan Godfrey

Institute of Neuroscience

Newcastle University

Clinical Ageing Research Unit

Campus for Ageing and Vitality

Newcastle upon Tyne,

NE4 5PL.

Email: alan.godfrey@ncl.ac.uk

Tel: +44 (0) 1912081245

Fax: +44 (0) 1912081251 


\begin{abstract}
(184/250)
Free-living ambulation with accelerometer-based devices is an attractive methodology to assess habitual behaviour within Parkinson's disease (PD). However, slowness of movement can contribute to difficulty in quantifying ambulatory/walking outcomes within this group by these devices. This study investigates the use of a commercial accelerometer device $\left(\operatorname{activPAL}^{\mathrm{TM}}\right)$ in those with moderate PD to understand its proprietary software (inbuilt algorithm) limitations. The values provided by the proprietary software are evaluated in comparison to novel algorithms on the same raw data to examine limitations for use within this cohort. The bespoke algorithms help to alter sensitivity in outcomes stemming from the same accelerometer data while also highlighting how slight changes in algorithms can drastically inflate/deflate values. In general, results show that the proprietary software generally quantifies lower values of outcomes (step and bout count), which is similar to previous findings. Variations in algorithm functionality highlight large heterogeneity in bout and step counts resulting from a lack of how they are defined within the literature. The novel alternative ambulatory algorithms presented here should be considered for use on raw data from the activPAL ${ }^{\mathrm{TM}}$ in those with moderate/severe PD.
\end{abstract}

Keywords: accelerometer; gait; bouts; free-living; habitual; algorithm; 


\section{Introduction}

Ambulatory activity (walking) is a useful measurement tool in neurodegenerative disorders, e.g. Parkinson's disease (PD) [1, 2]. Monitoring community-based activity may better inform diagnosis/treatment compared to traditional clinical assessment due to the habitual (free-living) movement [3-6]. Currently, accelerometer-based devices are primarily used to quantify timed periods of walking (bouts), number of bouts and steps accumulated during longitudinal monitoring (i.e. 7 days). Devices can be worn in a number of anatomical locations $[7,8]$ each generating a notable change in signal acceleration [9] and therefore requiring site specific analytical methods (algorithms). Typically devices are worn at or near the waist/lower back to examine whole body movement, but limitations in current algorithms prohibit robust segmentation of upright activity (standing and sedentary, i.e. sitting) with a device at that location and orientation [10].

An alternative attachment location is the upper thigh which easily segments between standing and sedentarism due to device placement and orientation $[11,12]$. However, this location is reliant on single limb movement potentially underestimating step count and bout definition during prolonged free-living assessment. Moreover, this could be exacerbated by rigid analytical methods (e.g. fixed thresholds) more suited to higher intensity ambulation rather than slow, less clearly defined walking as experienced within PD [13]. One commercial device worn on the thigh includes the activPAL ${ }^{\mathrm{TM}}$ which has been validated in young adults for bout length/count (at a resolution of 1 minute) during prolonged free-living activity [14] or scripted tasks [11]. However, large errors have been reported for estimated step count [10] from the same device during similar unstructured activity at slow speeds. To date no study has examined why the errors exist which may primarily stem from its front end proprietary software (internal algorithm). Nor has any study previously examined the utility of its raw accelerometer data for walking assessment in those with moderate/severe PD.

Therefore, the primary purpose of this study is to examine quantified bouts and step count from the proprietary software of the activPAL ${ }^{\mathrm{TM}}$ in those with moderate PD to robustly examine the device in those with slower less clearly defined walking. Secondary, values from the proprietary software were compared against suggested novel alternative algorithms used to investigate the suitability of the raw activPAL ${ }^{\mathrm{TM}}$ acceleration data and its applicability in quantifying $\mathrm{PD}$ ambulation/walking during 
free-living. We discuss all algorithms for use with raw activPAL ${ }^{\mathrm{TM}}$ data and make recommendations for future free-living PD walking assessment with this device.

\section{Algorithms}

\subsection{Participants}

PD participants 18 months post diagnosis were recruited from the ICICLE-GAIT study [3], a collaborative study with ICICLE-PD ${ }^{1}$ conducted between June 2009 and December 2011 [15]. The study was approved by the Newcastle and North Tyneside 1 Research Ethics Committee and Newcastle upon Tyne Hospitals NHS Foundation Trust (09/H0906/82). All participants gave their informed written consent. Demographics and motor-based clinical measures were recorded: selfreport freezing of gait (FOG), self-efficacy balance confidence scale (Activities Balance Self Confidence Scale, ABCs), severity of motor symptoms (Hoehn and Yahr scale, H\&Y) and section III (motor examination) of the modified Movement Disorder Society version of the Unified Parkinson's Disease Rating Scale (MDS-UPDRS III).

\subsection{Equipment}

The activPAL ${ }^{\mathrm{TM} 2}(53.0 \times 35.0 \times 7.0 \mathrm{~mm}, 20 \mathrm{~g}, 10 \mathrm{~Hz}, 8 \mathrm{bit}, \pm 2 \mathrm{~g})$ is a uni-axial accelerometer-based device with its output expressed as 64 analog to digital (A/D) units per $1 \mathrm{~g}$ (gravity, $1 \mathrm{~g}=9.81 \mathrm{~ms}^{-2}$, i.e. $1 \mathrm{~A} / \mathrm{D}$ unit equates to $16 \mathrm{mg}$ ). It was attached, in accordance with manufactures guidelines, to the midline of the upper thigh by a hydrogel adhesive (PALStickies ${ }^{3}$ ) and covered with Hypafix ${ }^{3}$ tape [16]. Participants were instructed to remove devices only during bathing and were provided with replacement adhesives and tape to re-attach each.

\subsection{Data processing}

Data from the device were downloaded via its graphical user interface program. Firstly, the program segmented, quantified and presented the data with outcomes according to its regular use in

\footnotetext{
${ }^{1}$ Incidence of Cognitive Impairment in Cohorts with Longitudinal Evaluation-Parkinson's disease

${ }^{2}$ PAL Technologies, Glasgow, UK

${ }^{3}$ BSN Medical Limited, Hull, UK
} 
an $\mathrm{Excel}^{4}$ (event) file estimating time spent sedentary (sitting/lying), standing, stepping and number of steps. Stepping data only in this format were analysed via MATLAB ${ }^{\circledR}$, as described previously (Program 1) [3, 17, 18], Fig. 1. Secondly, the software exported the raw accelerometer-based $\operatorname{activPAL}^{\mathrm{TM}}$ data to a comma separated value format (.csv). That raw format was processed by a bespoke MATLAB $^{\circledR}$ program (Program 2), implementing numerous variations on quantifying PD walking: importing the raw accelerometer data, identifying periods of upright from sitting/lying (sedentary), segmenting standing from stepping and quantifying walking outcomes. A representation of data analysis flow is presented in Fig. 1.

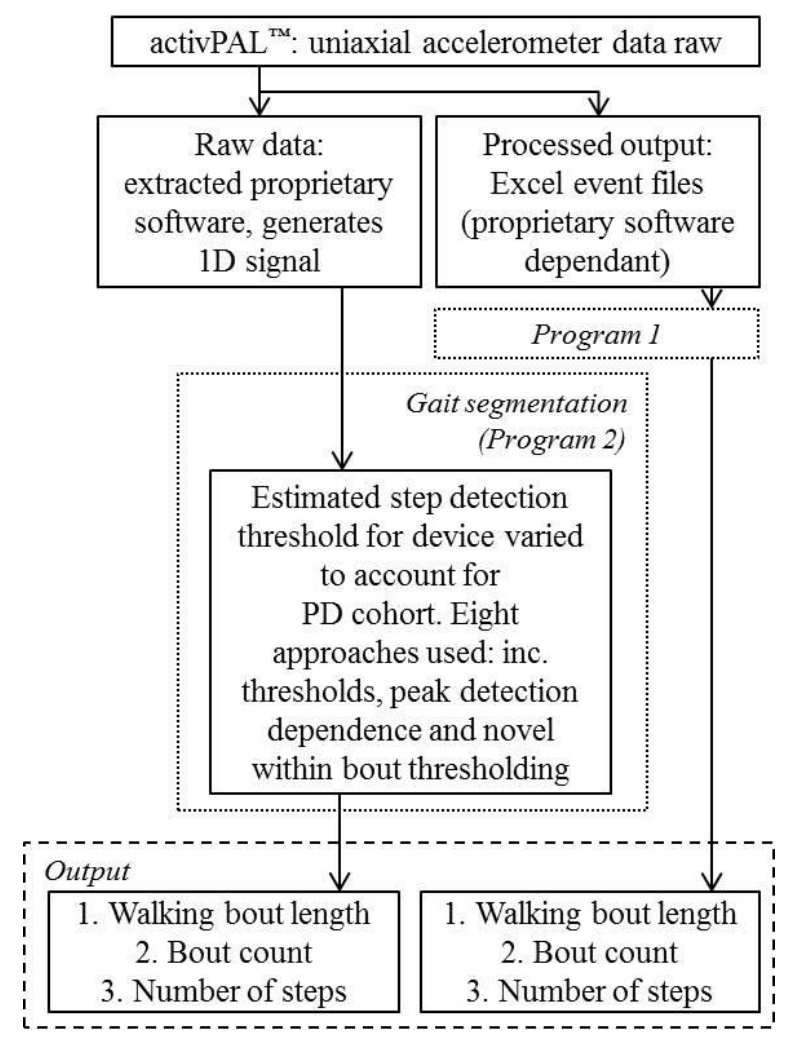

Figure 1: Data processing flow algorithm and analysis performed by the MATLAB $^{\circledR}$ programs.

\subsection{Alternate ambulatory algorithms}

Raw data were analysed utilising a number of algorithm configurations consisting of three stages: (i) segmenting sitting/lying (sedentary) from standing/walking (upright) by applying a predetermined threshold (value $=20 \mathrm{~A} / \mathrm{D}$ units, Fig. 2a\&b), (ii) applying a SD threshold (3.5 A/D units) of the complete bout to identify standing from walking and (iii) quantify stepping by considering a number of different configurations, below. All thresholds were chosen from visual observation based on the

\footnotetext{
${ }^{4}$ Microsoft Corp., Redmond, WA, USA
} 
mean value of the raw data while standing (approx. $150 \mathrm{AD}$ units) and lower/upper limits which may be sensitive to step detection (peaks), selected as $-20 /-25$ and $+20 /+25$, Fig. 2 .

1. Applying two random thresholds (Algorithm \#1 (150-25) = 125 and \#2 (150-20) = 130) on the negative peaks of a standing/stepping bout (Fig. 2c-i). Peaks (in all cases) were found using the MATLAB ${ }^{\circledR}$ findpeaks function, which had the potential to yield many peaks within the same step location. Thus, the 'MinPeakDistance' parameter (value $=8$ samples or $800 \mathrm{~ms}$ based on device sampling rate) within the function was implemented only here to eliminated smaller peaks within close proximity to a 'step' (i.e. true negative peak).

2. Positive/negative peak detection criteria to quantify a step. Based on the location of the device, a step was defined in the order of a forward (positive, green circle) and backward (negative, red circle) swing within the signal (Fig. 2c) based on positive/negative peak thresholds (Algorithm \#3=125/175 and \#4 = 130/170).

3. Positive/negative peak detection criteria to quantify a step excluding 1 single step. This method (algorithm \#5) is similar to that in \#3 (125/175), but excluded a bout with a single step (e.g. Fig. 2c i-ii - d). This was implemented to remove what may be termed a nonpurposeful step/walk or single step in a fixed location.

4. Positive/negative peak detection criteria to quantify a step based on a within bout adaptable threshold (algorithm \#6). This novel method estimated the mean and SD per individual stepping bout to define a peak threshold for that bout (mean of the bout \pm SD of the same bout). The SD was estimated from all values of the bout >100 to account for very large estimations based on a brief period (1-2s) of crouching/kneeling that may occur: manual inspections of the signals showed brief periods where the general trend of the signal approached the sedentary/upright threshold (i.e. 20 A/D units), perhaps due to picking an object from the floor. Removal of continuous values below 100 ensured the SD value remained small to detect smaller peak missed by previous methods, Fig. 2c-iii. Thus, individual (unique) mean and SD values were calculated for each bout, enabling an adaptable threshold to be applied specific to each bout of walking. This may be particularly useful for 
ensuring accurate step quantification due to slowness of walking speed in older adult during different conditions or pathological populations with varying/deteriorating walking ability.

5. It has been shown that the proprietary software for the device applies an internal threshold between stepping bouts that group bouts before/after a brief bout of standing, i.e. a maximum resting period (MRP) of 6s [19]. The above Algorithms were implemented with the same 6s methodology, but Algorithms \#7 and \#8 reduced this to $3 \mathrm{~s}$ and $1 \mathrm{~s}$, respectively. (The SD thresholding method per bout (Algorithm 6, above) was used here).

Bout length (period of time stepping/walking) was calculated from the time of the first step to the last step based on the aforementioned algorithms with the addition of a 1.6s buffer to account for step initiation/termination. This method was based upon inspection of the raw data and parallels drawn to the outcomes from the proprietary software. 
(a)

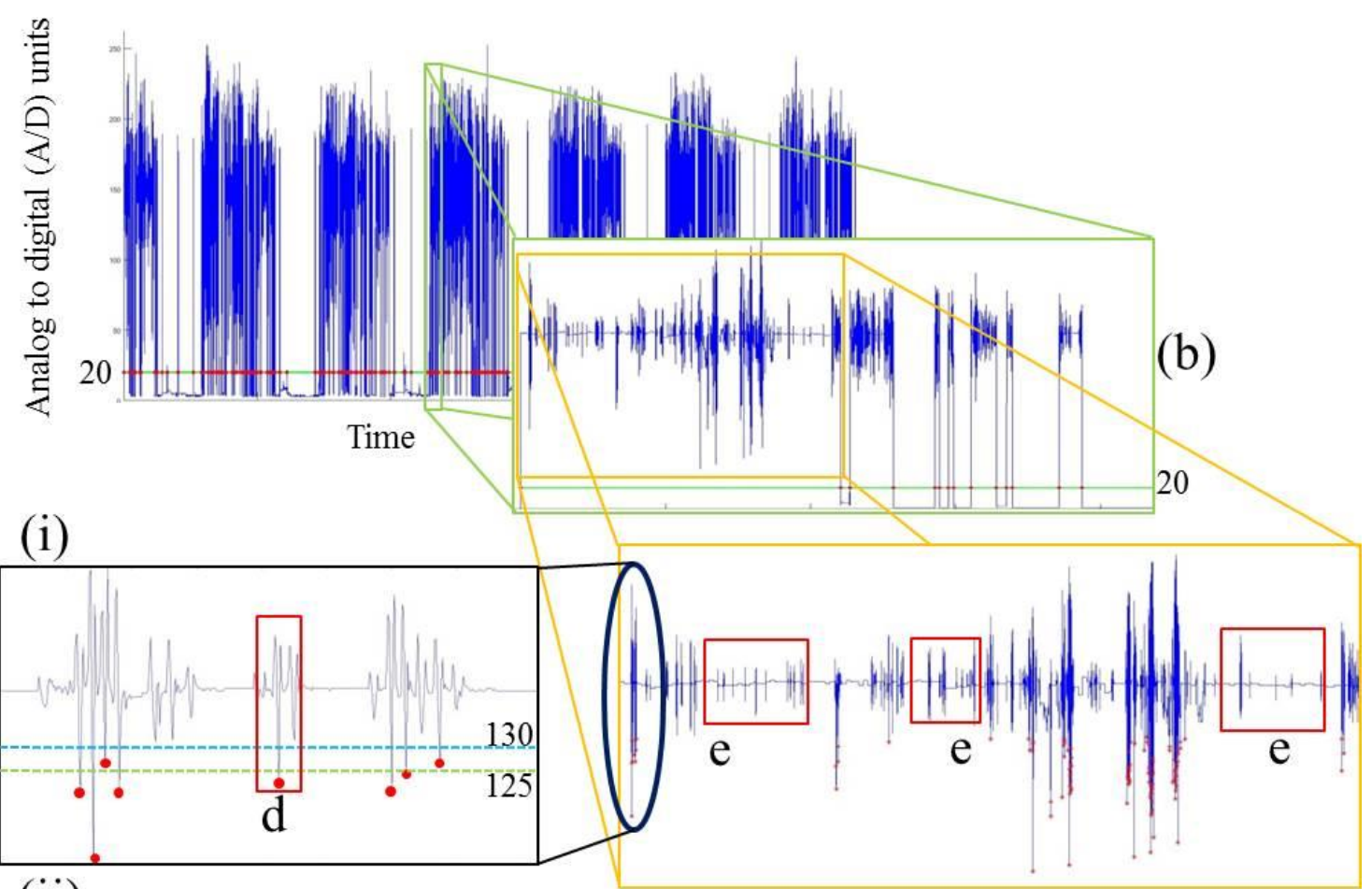

(ii)
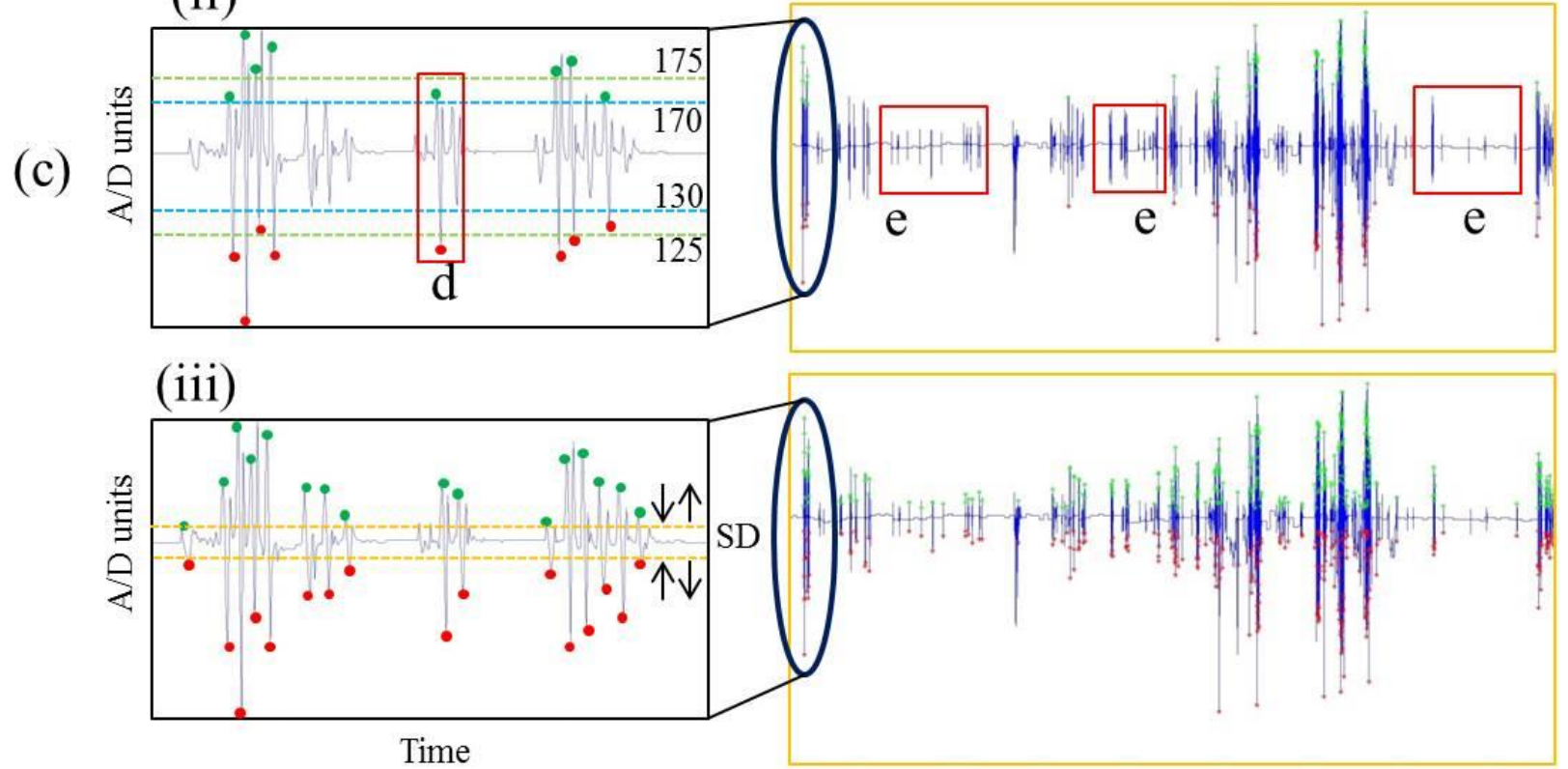

Figure 2:

a. Raw free-living uniaxial accelerometer for 7 days

b. Zoomed data of sitting/lying and standing/stepping bouts based on the orientation and variations within the signal, respectively

c. Zoomed standing/stepping bout (right) and enlarged examples of step detection based on Algorithm \#1 (i), Algorithm \#3 (ii) and Algorithm \#6/7/8 (iii). (Note: The portion of this signal corresponds to Fig 3).

d. Elimination of a one-step bout, algorithm \#5.

e. Possible bouts of walking/stepping and steps unquantified by the proprietary software and algorithms \#1-5, yet identified by algorithm \#6/7/8. 


\section{Results}

Over 55.5 days (approx. 80000 minutes) were recorded, the device failed to record for just over half a day on one occasion. Participants $(n=8)$ demographic, clinical and cognitive descriptors are shown in Table 1. All participants reported no FOG. Compared to reference [20] control data (shown in italics), participants presented with slightly lower confidence in their balance (ABCs: $86.2 \mathrm{v} 91.3$ ). All participants presented with H\&Y II with a mean score of 27.9 on the MDS-UPDRS III, symptomatic of moderate PD [3].

Table 1: Participant demographic and clinical characteristics

\begin{tabular}{lc}
\hline Characteristic & Mean \pm SD \\
\hline M/F (n) & $4 / 4$ \\
Age (years) & $68.0 \pm 8.4$ \\
Height (m) & $1.7 \pm 0.1$ \\
BMI $\left(\mathrm{Kg} / \mathrm{m}^{2}\right)$ & $26.0 \pm 4.3$ \\
\hline ABCs $(0-100 \%)$ & $86.2 \pm 15.6$ \\
\hline Hoehn \& Yahr (n) & H\&Y I - 0 \\
\multicolumn{2}{l}{ H\&Y II - 8 } \\
\hline MDS-UPDRS III & H\&Y III - 0 \\
\hline BMI: body mass index, ABCs: activities specific \\
balance confidence scale, UPDRS: Unified \\
Parkinson's Disease Rating Scale
\end{tabular}

There was a $128 \%$ increase in total time walked from (proprietary) 523.3mins/week to (algorithm \#2) 1198.1mins/week depending on algorithm configuration, Table 2. Slight fixed thresholding variation (125 vs. 130 and 130/170 vs. 125/175) increased total walking time between 124.8 (454.3329.5 ) to 200.3 (1198.1-997.8) mins/week, (or up to 30mins/day), respectively.

Bout length times (ranges) were comparable and consistent except for Algorithms \#3, \#5 and \#8 suggesting peak thresholds (125/175) and a MRP gap of 1s between bouts for this population are unsuitable. Moreover, if allowing for a MRP between bouts, the value/threshold should be in excess of $1 \mathrm{~s}$ (i.e. 1 stride) accounting for device location and inter limb dependency to define ambulation/walking. Bout count almost doubled (2955 to 5256) due to the detection of larger number of shorter bouts with the increased sensitivity in possible step detection.

Algorithm variations resulted in a 79\% increase in steps detection within the device (proprietary: 19,041 to algorithm \#2: 34,126) due to an increased sensitivity approach within the same signal, Table 2 and Fig. 2c. However, it was noted that Algorithm \#1 and \#2 (fixed negative thresholding) resulted 
in a possible 'false' steps due to what appeared to be brief periods of couching/kneeling and hence change in orientation of the device (trend in acceleration signal) dropping acceleration below the sedentary/upright threshold.

$<$ Table 2, end of document >

\section{Discussion}

The purpose of this study was to investigate the use of the activPAL ${ }^{\mathrm{TM}}$ to quantify slower, reduced quality of ambulation/walking as experience in those with moderate PD. For the purposes of this study, bout and step outcomes from its proprietary software were compared to other novel algorithms suggested for use on the raw accelerations of the same device. This was a novel aspect of the study and to the authors knowledge no previous work has investigated or suggested alternative algorithms for the raw activPAL ${ }^{\mathrm{TM}}$ data. Bout length (range) was consistent for 6 of the algorithms (inc. proprietary software), yet bout and step count varied greatly throughout prompting an urgent need to clarify the definition of ambulation in free-living conditions, especially in PD where clarity of movement is less clear/distinctive. Moreover, this highlights the weakness of utilising fixed thresholds when pragmatically deploying devices across a range of walking abilities. However, the raw data captured by the activPAL ${ }^{\mathrm{TM}}$ has utility to quantify ambulation in moderate/severely affected PD by applying the alternate algorithm(s) suggested here rather than accepting values from the proprietary software which may underestimate.

\section{Bout length}

Applying alternative algorithms to the device resulted in the identification and increased length and number of possible gait bouts (Fig 2e and Fig. 3A), Table 2. Yet, values (maximum range) were similar to those generated by the proprietary software. Previous studies have evaluated the bout length properties of the device to other accelerometer-based equipment at 1 minute epochs or visual inspection $[11,14]$ with good accuracy, but never in free-living older adults with PD. However, the similarities between bout lengths here suggest algorithms are stable and comparable for defining bout length in PD. 
Algorithm \#6 (7 and 8) seems the most logical for future use when considering the functionality of the device, placement on the thigh and patient cohort: true step identification due to the reliance on positive/negative swings in the acceleration signal and adaptable threshold to account for any change in speed/intensity due to fatigue or accompanied walking. Notable areas of activity (possible gait initiation) were identified (Fig. 3, gold circles C) which could impact the length of a bout (total walking time, number of steps included) but were not detected by the algorithms presented here, even when using a variable within bout thresholding method (i.e. use of SD rather than predetermined values of $120 / 170$ and 125/175). Sensitivity could be increased by altering the first SD threshold (stage ii) to segment standing from stepping. For more advanced PD (H\&Y III) this could possibly be revised down (e.g. 2.5) to account for even slower ambulation/walking acceleration profiles. Additionally, although self-efficacy FOG wasn't reported, quantification of these regions may be useful in capturing and exploring quality of initiation, termination or festination of walking with these raw data.

\section{Bout count (and total time)}

The proprietary software of the device often failed to quantify short or slower less intense bouts, which also impacted total (accumulated) ambulatory time, Fig. 2e or 3C. Three bouts are highlighted, yet only 2 were recognised by the proprietary software denoted by the green (start) and red (end) lines. However, the centre bout (Fig. 3B) and step (Fig. 3D) closely resemble a short bout that went unrecognised, which were common occurrences from manual visual inspection of all participant signals. However, alternative algorithms increased bout count to include those depicted in Fig 2e, Fig 3A. These included the variations within the MRP (1, 3 and $6 \mathrm{~s})$ between recognised walking bouts which has been previously investigated and inherent within this device [19]. This influenced bout count as well as total time and should be considered within moderate/severe PD due to functional ability and/or fatigue. However, clear definitions on what defines (i) an individual walking bout or (ii) separate bouts from another due to any length of upright/standing resting period, is yet to be ratified within the literature and should be considered in future walking recognition algorithms. 


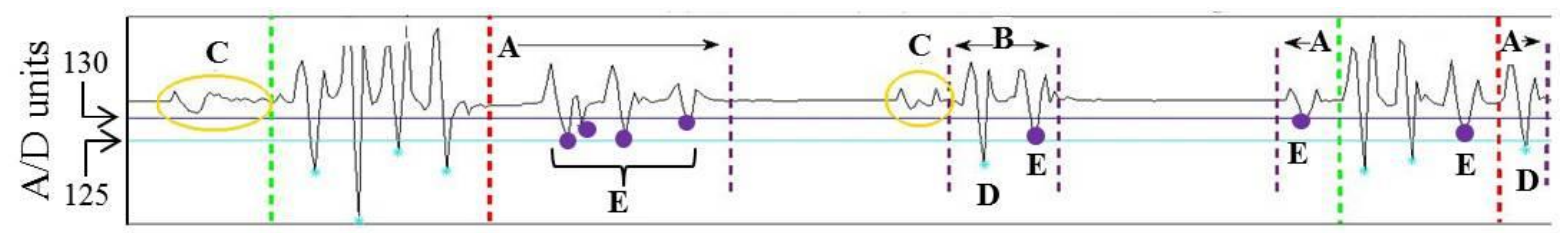

Time

Figure 3:

The same portion of the raw accelerometer signal corresponding to Fig $2 \mathrm{c}$. Two detected bouts with start/end times determined by its proprietary software and inbuilt threshold, total number of detected steps $=6$ (between vertical green/red lines). Applying two different algorithms (\#1 and \#2) results in: increased bout lengths (A), detection of a new bout (B) and an increased number of steps (D, lilac, threshold of $125(\# 1): n=8)$, or (E, purple, threshold of $130(\# 2): n=15)$.

- Note \#1: the proprietary software failed to detect a single (possible) step within the area of interest $\mathrm{B}$, but was classified (step/bout) by some of suggested methodology, where appropriate.

- $\quad$ Note \#2: The areas of interest C (gold circles) not quantified as steps by any of the algorithms which may be useful for examining poor gait initiation in this cohort.

\section{Step count}

While the exact functionality of the activPAL ${ }^{\mathrm{TM}}$ proprietary software is unknown, its step accuracy were recently shown to a lower number of step count during scripted and outdoor use under different speeds (slow and fast) in different ages [10,21]. The alternate algorithms presented in this study may help in quantifying steps in those with moderate/severe motor disorders from the raw data. While inclusion of less purposeful steps (i.e. shuffling) creates an abundance of possible data to examine gait, it can blur the lines between ad-hoc/sporadic gait (e.g. lateral movement due to cooking within a kitchen), reduced clarity of walking due to cognitive loading (e.g. dual tasking) and continuous/purposeful walking. Moreover, defining steps within or between cohorts need to be considered for the population in question. The quantification of steps during sporadic walking within PD may be more informative and abundant [22] than those accumulated during a few continuous bouts, more representative of ability rather than disease severity. Participants within this study presented moderate symptoms (H\&Y II, MDS-UPDRS III 27.9) and could be described as active but having some degree of motor impairment suggesting that the commercial algorithm to quantify their steps (and bouts) as inadequate. This is supported by the proposed alternative methods of step detection form the same device (Algorithms \#1, 2, 6-8) where a slight change of threshold or application of a novel adaptable threshold per bout resulted in increased step count. 


\section{Advantages and limitations of proposed algorithms}

The proposed methodology of positive/negative peak reliance to define a step based on device location (thigh) seemed logical to best represent the functional mechanics of walking. Moreover, the novel within bout threshold to account for stepping performance (in that bout of walking) seemed equally logical to account for variations in gait under different environmental conditions, improved step detection due to accompanied walks and/or dual tasking. However, ambulation is presented here in the general context (all walking) and in reality extends to more levels of complexity, such as stair ascent/descent and walking on uneven terrain which may negatively impact step count based upon the applied methodology assuming level walking [10].

\section{Conclusion}

Comparable bout length times were found across all algorithms suggesting its use as a robust outcome from the device. Yet it was possible to generate large variation in bout and step count from the same acceleration data, stemming from short bouts and slight variations in methods. Thorough investigation of the data found that numerous ambulatory/walking events were unaccounted for which impacted algorithm agreement. However, clear definition of walking events (definition of a step and start/end of a bout) from free-living data needs to be ratified within the literature enabling more clarified habitual walking assessment. It has been shown that the activPAL ${ }^{\mathrm{TM}}$ commercial software may be unable to account for all bout and step count in moderate PD due to reduced clarity of movement with its values alluding to more purposeful walking. Yet, examination of the same raw data enables the researcher to apply alternate, more stringent and novel methodologies (like those proposed here) and should be considered with future use of the device within moderate/severe PD. All methodologies presented here should be validated to a gold standard video/observer and constitute ongoing work.

\section{Ethical Approval}

The study was approved by the Newcastle and North Tyneside 1 Research Ethics Committee and Newcastle upon Tyne Hospitals NHS Foundation Trust (09/H0906/82). 


\section{Acknowledgements}

The authors would like to thank Ms Dadirayi Mhiripiri for her help with participant recruitment and data collection and Mr. Philip Brown for cohort data checking. The authors would also like to thank the participants who volunteered for this study. AG is supported by the National Institute for Health Research (NIHR) Newcastle Biomedical Research Centre (BRC) and Unit (BRU) based at Newcastle upon Tyne Hospitals NHS Foundation Trust and Newcastle University. The work was also supported by the NIHR Newcastle Clinical Research Faculty infrastructure funding. The views expressed are those of the authors and not necessarily those of the funders. ICICLE-PD is supported by Parkinson's UK.

\section{References}

[1] S. Lord, B. Galna, and L. Rochester, "Moving forward on gait measurement: toward a more refined approach," Mov Disord, vol. 28, pp. 1534-43, Sep 152013.

[2] A. Salarian, H. Russmann, F. J. G. Vingerhoets, P. R. Burkhard, and K. Aminian, "Ambulatory Monitoring of Physical Activities in Patients With Parkinson's Disease," IEEE Transactions on Biomedical Engineering, vol. 54, pp. 2296-2299, 2007.

[3] S. Lord, A. Godfrey, B. Galna, D. Mhiripiri, D. Burn, and L. Rochester, "Ambulatory activity in incident Parkinson's: more than meets the eye?," J Neurol, vol. 260, pp. 2964-72, Dec 2013.

[4] K. S. van Schooten, M. Pijnappels, S. M. Rispens, P. J. Elders, P. Lips, and J. H. van Dieen, "Ambulatory fall-risk assessment: amount and quality of daily-life gait predict falls in older adults," J Gerontol A Biol Sci Med Sci, vol. 70, pp. 608-15, May 2015.

[5] K. S. van Schooten, S. M. Rispens, P. J. Elders, P. Lips, J. H. van Dieen, and M. Pijnappels, "Assessing physical activity in older adults: required days of trunk accelerometer measurements for reliable estimation," J Aging Phys Act, vol. 23, pp. 9-17, Jan 2015.

[6] A. Mirelman, N. Giladi, and J. M. Hausdorff, "Body-Fixed Sensors for Parkinson Disease," JAMA, vol. 314, pp. 873-4, Sep 12015.

[7] A. Godfrey, R. Conway, D. Meagher, and O. L. G, "Direct measurement of human movement by accelerometry," Med Eng Phys, vol. 30, pp. 1364-86, Dec 2008.

[8] M. J. Mathie, A. C. Coster, N. H. Lovell, and B. G. Celler, "Accelerometry: providing an integrated, practical method for long-term, ambulatory monitoring of human movement," Physiol Meas, vol. 25, pp. R1-20, Apr 2004.

[9] I. Cleland, B. Kikhia, C. Nugent, A. Boytsov, J. Hallberg, K. Synnes, et al., "Optimal placement of accelerometers for the detection of everyday activities," Sensors (Basel), vol. 13, pp. 9183-200, 2013.

[10] F. A. Storm, B. W. Heller, and C. Mazza, "Step Detection and Activity Recognition Accuracy of Seven Physical Activity Monitors," Plos One, vol. 10, Mar 192015.

[11] P. M. Grant, C. G. Ryan, W. W. Tigbe, and M. H. Granat, "The validation of a novel activity monitor in the measurement of posture and motion during everyday activities," Br J Sports Med, vol. 40, pp. 992-7, Dec 2006. 
[12] C. G. Ryan, P. M. Dall, M. H. Granat, and P. M. Grant, "Sitting patterns at work: objective measurement of adherence to current recommendations," Ergonomics, vol. 54, pp. 531-538, 2011/06/01 2011.

[13] M. E. Morris, R. Iansek, T. A. Matyas, and J. J. Summers, "The pathogenesis of gait hypokinesia in Parkinson's disease," Brain, vol. 117, pp. 1169-1181, 1994-10-01 00:00:00 1994.

[14] A. Godfrey, K. M. Culhane, and G. M. Lyons, "Comparison of the performance of the activPAL Professional physical activity logger to a discrete accelerometer-based activity monitor," Med Eng Phys, vol. 29, pp. 930-4, Oct 2007.

[15] T. K. Khoo, A. J. Yarnall, G. W. Duncan, S. Coleman, J. T. O'Brien, D. J. Brooks, et al., "The spectrum of nonmotor symptoms in early Parkinson disease," Neurology, vol. 80, pp. 276-81, Jan 152013.

[16] P. M. Grant, P. M. Dall, S. L. Mitchell, and M. H. Granat, "Activity-monitor accuracy in measuring step number and cadence in community-dwelling older adults," J Aging Phys Act, vol. 16, pp. 201-14, Apr 2008.

[17] A. Godfrey, S. Lord, B. Galna, J. C. Mathers, D. J. Burn, and L. Rochester, "The association between retirement and age on physical activity in older adults," Age Ageing, vol. 43, pp. 38693, May 2014.

[18] K. Mactier, S. Lord, A. Godfrey, D. Burn, and L. Rochester, "The relationship between real world ambulatory activity and falls in incident Parkinson's disease: influence of classification scheme," Parkinsonism Relat Disord, vol. 21, pp. 236-42, Mar 2015.

[19] G. Barry, B. Galna, S. Lord, L. Rochester, and A. Godfrey, "Defining ambulatory bouts in free-living activity: Impact of brief stationary periods on bout metrics," Gait \& Posture, vol. In Press, 2015.

[20] S. Del Din, A. Godfrey, and L. Rochester, "Validation of an accelerometer to quantify a comprehensive battery of gait characteristics in healthy older adults and Parkinson's disease: toward clinical and at home use," IEEE J Biomed Health Inform, Apr 22015.

[21] S. Aminian and E. A. Hinckson, "Examining the validity of the ActivPAL monitor in measuring posture and ambulatory movement in children," The International Journal of Behavioral Nutrition and Physical Activity, vol. 9, pp. 119-119, 2012.

[22] M. S. Orendurff, J. A. Schoen, G. C. Bernatz, A. D. Segal, and G. K. Klute, "How humans walk: bout duration, steps per bout, and rest duration," J Rehabil Res Dev, vol. 45, pp. 1077 89, 2008. 
Tables

Table 2: Median (Range) of walking/stepping outcomes for 7 days with different algorithm configurations

\begin{tabular}{|c|c|c|c|c|}
\hline Configuration & Total time (mins) & Bout length (mins) & Bout count & Step count \\
\hline Proprietary software & $523.3(254.9-807.6)$ & $0.1(0.0-21.0)$ & $2955(1580-4051)$ & $19041(8748-30112)$ \\
\hline Algorithm \#1: negative peak threshold (125) & $997.8(514.5-1335.2)$ & $0.1(0.1-24.8)$ & $3857(2161-5097)$ & $27624(14125-40688)$ \\
\hline Algorithm \#2: negative peak threshold (130) & $1198.1(569.2-1462.2)$ & $0.1(0.1-24.8)$ & $3878(2151-5316)$ & $34126(16223-44444)$ \\
\hline Algorithm \#3: +/- peak dependency $(125 / 175)$ & $329.5(123.9-602.4)$ & $0.1(0.1-9.0)$ & $1794(872-3328)$ & $10906(3548-20517)$ \\
\hline Algorithm \#4: +/- peak dependency (130/170) & $454.3(221.6-796.1)$ & $0.1(0.1-20.9)$ & $2163(1357-3802)$ & $14725(6620-26388)$ \\
\hline Algorithm \#5: +/- peak dependency $(125 / 175)$ - 1 step bout & $301.7(101.2-512.6)$ & $0.1(0.1-9.0)$ & $1394(690-2346)$ & $10506(3205-19931)$ \\
\hline Algorithm \#6: +/- peak dependency (SD threshold), 6s gap & $858.3(446.0-1276.4)$ & $0.2(0.1-20.9)$ & $3342(1613-5304)$ & $29047(15767-40140)$ \\
\hline Algorithm \#7: +/- peak dependency (SD threshold), 3s gap & $795.6(410.0-1171.1)$ & $0.1(0.1-20.7)$ & $4301(2106-6512)$ & $29034(15767-40136)$ \\
\hline Algorithm \#8: +/- peak dependency (SD threshold), 1s gap & $765.0(392.6-1127.6)$ & $0.1(0.1-13.0)$ & $5256(2634-7810)$ & $29034(15767-40136)$ \\
\hline
\end{tabular}

